\title{
Topographic Maps of Visual Spatial Attention in Human Parietal Cortex
}

\author{
Michael A. Silver, ${ }^{1}$ David Ress, ${ }^{1}$ and David J. Heeger ${ }^{1,2}$ \\ ${ }^{1}$ Department of Psychology, Stanford University, Stanford, California; and ${ }^{2}$ Department of Psychology and Center for Neural Science, \\ New York University, New York, New York
}

Submitted 21 December 2004; accepted in final form 1 April 2005

\begin{abstract}
Silver, Michael A., David Ress, and David J. Heeger. Topographic maps of visual spatial attention in human parietal cortex. J Neurophysiol 94: 1358-1371, 2005. First published April 7, 2005; doi:10.1152/jn.01316.2004. Functional magnetic resonance imaging (fMRI) was used to measure activity in human parietal cortex during performance of a visual detection task in which the focus of attention systematically traversed the visual field. Critically, the stimuli were identical on all trials (except for slight contrast changes in a fully randomized selection of the target locations) whereas only the cued location varied. Traveling waves of activity were observed in posterior parietal cortex consistent with shifts in covert attention in the absence of eye movements. The temporal phase of the fMRI signal in each voxel indicated the corresponding visual field location. Visualization of the distribution of temporal phases on a flattened representation of parietal cortex revealed at least two distinct topographically organized cortical areas within the intraparietal sulcus (IPS), each representing the contralateral visual field. Two cortical areas were proposed based on this topographic organization, which we refer to as IPS1 and IPS2 to indicate their locations within the IPS. This nomenclature is neutral with respect to possible homologies with wellestablished cortical areas in the monkey brain. The two proposed cortical areas exhibited relatively little response to passive visual stimulation in comparison with early visual areas. These results provide evidence for multiple topographic maps in human parietal cortex.
\end{abstract}

\section{N T R O D U C T I O N}

Presentation of a cue that directs attention to the location of a stimulus enhances detection of the stimulus, even in the absence of overt eye movements (Bashinski and Bacharach 1980; Pashler 1998; Posner 1980). Posterior parietal cortex (PPC) has been implicated in the control of visual spatial attention in studies using a variety of techniques, including electrophysiology (Colby and Goldberg 1999), neuropsychology (Mesulam et al. 1999), and neuroimaging (Corbetta and Shulman 2002). Top-down attention control signals modulate activity in visual cortical areas in a retinotopically specific manner (Brefczynski and DeYoe 1999; Gandhi et al. 1999; Kastner et al. 1999; McMains and Somers 2004; Ress et al. 2000; Tootell et al. 1998). That is, the attention signals are evident only in the portions of each visual area that represent the attended region of the visual field. The sources of these top-down attention signals are not known, but it seems likely that the proximal sources will include areas in PPC. Because attention is spatially selective, these areas are likely to exhibit topographic representations of the visual field.

Topographic mapping with functional magnetic resonance imaging (fMRI) has been used to define a number of visual

\footnotetext{
Address for reprint requests and other correspondence: M. A. Silver; Helen Wills Neuroscience Institute; University of California, Berkeley; 132 Barker Hall, \#3190; Berkeley, CA 94720-3190 (E-mail: masilver@berkeley.edu).
}

cortical areas (DeYoe et al. 1996; Engel et al. 1994, 1997; Press et al. 2001; Sereno et al. 1995). Sereno et al. (2001) used topographic mapping to identify an area in human PPC that contained a representation of the contralateral visual field. In their experiment, a visual target was briefly presented, and subjects had to remember its location for several seconds. By presenting the target at a sequence of positions during a series of trials, Sereno et al. observed a traveling wave of activity across a region of PPC, indicating that this area was topographically organized. In addition, the companion paper (Schluppeck et al. 2005) to the present study identified additional topographic maps in human parietal cortex based on responses during a delayed saccade task similar to that used by Sereno et al. (2001).

We performed a complementary series of experiments to examine topographic organization during a spatial attention task, in the absence of eye movements. In our experiments, attention was systematically directed in sequence to a series of locations around a central fixation point. Each trial began with a cue that directed subjects to attend one of the target locations. After a randomized delay period, low-contrast grating patches were presented at some of the target locations (50\% probability, independently for each location). Subjects were instructed to maintain fixation and to indicate whether a target was presented at the cued location. Because the cued location varied systematically from trial to trial, we expected a linear relationship between the temporal phase of measured fMRI responses and angular locations in the visual field.

We found clear evidence of topographically organized regions in the parietal cortex. The locations of phase reversals in the maps are consistent with the existence of at least two distinct topographic areas. We propose to name these cortical areas IPS1 and IPS2, based on their location within the intraparietal sulcus, although alternative interpretations involving further subdivisions of these areas are also possible [see DISCUSSION and the companion paper (Schluppeck et al. 2005)]. The two areas were adjacent to one another, and one of them (IPS1) was adjacent and just anterior to visual area V7. The boundaries between V7 and IPS1 and between IPS1 and IPS2 were defined by phase reversals, similar to those that define the boundaries between early visual cortical areas. Although these areas clearly responded when attention was covertly allocated, their responses to passive visual stimulation were much weaker than those in early visual areas.

The experiments described here were aimed at identifying cortical areas and characterizing their topography. They served only to define the cortical areas, not to reveal their function.

The costs of publication of this article were defrayed in part by the payment of page charges. The article must therefore be hereby marked "advertisement" in accordance with 18 U.S.C. Section 1734 solely to indicate this fact. 
We describe the IPS regions as containing topographic maps of sensory attention because they were defined using a spatial attention task. We leave open the possibility, however, that these cortical areas may also (or instead) be involved in covert motor intention (Andersen and Buneo 2002). Some of these data have been published previously in abstract form (Silver et al. 2004a).

\section{METHODS}

\section{Subjects}

The experiments were undertaken with the written consent of the subjects and in compliance with the safety guidelines for MR research. The experimental protocol was approved by the human subjects Institutional Review Boards at Stanford University and at New York University.

Five subjects participated in the study, all of whom had extensive experience as subjects in psychophysical and fMRI experiments. In particular, they had all served previously as subjects in fMRI studies that involved performing psychophysical discrimination tasks in the scanner while maintaining eye fixation. Subjects practiced the task for several hours outside the scanner until their psychophysical thresholds reached asymptotic levels of performance. This allowed precise determination of their thresholds and ensured that all fMRI data were acquired after their performance had stabilized. Subjects received feedback on every trial during the practice sessions, but no feedback was provided during the fMRI sessions. Each subject participated in at least 4 fMRI scanning sessions: one to obtain high-resolution anatomical images, at least one standard retinotopic mapping session to identify the early visual cortical areas, and between 2 and 6 sessions to identify topographic attention maps.

One subject was excluded from the study because a majority of his scans were contaminated by imaging artifacts. One of the artifacts was due to the fact that the subject's superior sagittal sinus was close to the edge of the field of view. This artifact was not evident in the other 4 subjects, presumably because of individual differences in the anatomy of the superior sagittal sinus. For this one subject, the large pulsatile signal from the sinus was mislocalized to other parts of the field of view, including PPC. Of those scans that did not contain this pulsatility artifact (which were acquired using echo planar instead of spiral imaging), many were contaminated by gross head motion for which post hoc motion correction failed to compensate. Overall, 51 out of 75 scans had to be excluded because of obvious pulsatility or motion artifacts. Analysis of the remaining scans replicated the results observed in the other subjects, but the signal variability was much higher, presumably as a result of the presence of residual artifacts.

\section{Presentation of visual stimuli}

Stimuli were presented using a flat-panel LCD monitor (MultiSync 2000; NEC, Itasca, IL) that was encased in a Faraday cage with an electrically conductive glass front window. The monitor was placed at the end of the magnet bore nearest the subjects' heads, and images were viewed through an angled mirror positioned above the subjects' eyes. The refresh rate of the monitor was $67 \mathrm{~Hz}$ for Stanford experiments and $60 \mathrm{~Hz}$ for New York University experiments.

\section{Attention-mapping protocol}

The visual stimuli and task were designed to evoke traveling waves of cortical activity in areas where attention signals were topographically organized (Fig. 1). Subjects were instructed to maintain fixation while attending and responding to a sequence of cued locations in an annulus surrounding the fixation point. Each approximately 5-min scan consisted of either 6 or 8 cycles, in which each cycle represented one complete traversal of the visual field by the focus of attention. A single cycle consisted of either 8 or 12 trials. The focus of attention moved either in a clockwise (CW scans) or counterclockwise (CCW scans) direction from trial to trial, so attention was periodically directed to each visual field location within the annulus for one trial of each cycle. The annulus containing the cued locations was defined by two black circles (inner diameter $1.5^{\circ}$, outer diameter $4.5^{\circ}$ ) and was subdivided into either 8 or 12 segments. The segments and a $0.125^{\circ}$ square central fixation point were continuously presented on a neutral gray background. A single segment was cued at the beginning of each trial by an electronically synthesized verbal cue ("one," "two," "three," etc.), and subjects covertly directed their attention to the designated segment.

Following a delay period of variable and randomized duration (250 to $1,750 \mathrm{~ms}$ ), a target was presented in the cued segment on $50 \%$ of the trials. Because the time of target presentation was not the same on every trial, the subjects had to actively attend to the cued segment for a significant portion of each trial. Regardless of the length of the delay period, an auditory click was presented 2,375 ms after the beginning of the trial. This click indicated that subjects should respond with a button press to indicate whether they perceived a target in the cued segment. The subjects had a $625-\mathrm{ms}$ period to respond, and at the end of this response period, the verbal cue initiating the next trial was presented. Thus the total trial duration was always $3 \mathrm{~s}$.

We confirmed in a separate series of psychophysical experiments, using a similar experimental protocol, that attentional selection was required for optimal performance in this task. Specifically, performance (as indexed by contrast thresholds for target detection) was improved when subjects were provided with a spatially informative cue compared with a very similar task in which they had to distribute their attention over a larger portion of the visual field (Silver et al. 2004b).

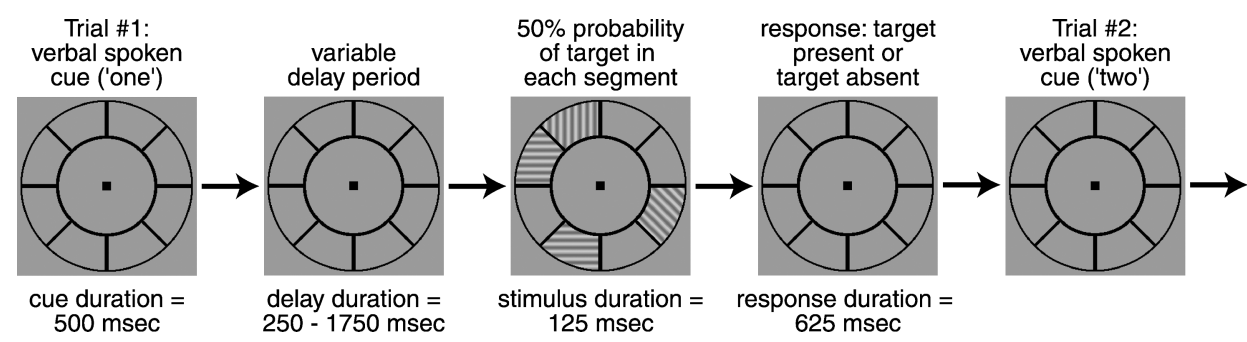

FIG. 1. Covert attention-mapping task used to generate maps of attention-related activity. Each trial began with an electronically synthesized spoken cue that corresponded to one of the segments in the annulus. After a variable delay period, a grating was presented with $50 \%$ probability, independently in each segment. An auditory click then signaled subjects to respond with a button press indicating whether a target was present or absent in the cued segment. After the response, the next trial began with another verbal cue that directed attention to one of the adjacent segments, either ascending in number (clockwise scans) or descending (counterclockwise scans). Total trial duration was $3 \mathrm{~s}$. Subjects were instructed to maintain fixation throughout each scan and responded only to the cued segment on each trial. Only the cued segment varied systematically over time. Although there were visual stimuli presented on every trial, there was no systematic pattern of visual stimulation. 
TABLE 1. Psychophysical sensitivities for target detection during attention mapping

\begin{tabular}{ccc}
\hline \hline Subject & Contrast Range (\%) & $d^{\prime}$ \\
\hline MAS & $1.75-2.6$ & 0.58 \\
JM & $3.2-3.4$ & 1.33 \\
DBR & 3.0 & 0.88 \\
DJH & $3.0-4.0$ & 1.01 \\
\hline
\end{tabular}

The target consisted of a 4 cyc/deg sine-wave grating, presented for $125 \mathrm{~ms}$. The target contrast was ramped on and off smoothly (contrast-modulated by one half cycle of a 4-Hz temporal sinusoid). Target contrast was very low (Table 1) and corresponded to each subject's detection threshold $\left(d^{\prime} \approx 1\right)$. Even though the threshold contrast was determined separately for each subject in practice sessions before scanning, performance varied slightly from session to session. Behavioral data were therefore collected and analyzed in real time during scanning sessions, and the target contrast was sometimes adjusted between scans to maintain $d^{\prime} \approx 1$ (Table 1 reports the range of contrasts actually used during scanning). The response amplitudes evoked by these barely detectable low-contrast stimuli are small $(<0.1 \%$ change in image intensity; Ress and Heeger 2003), much smaller than the range of responses that can be evoked by variability in attention (about 1\% change in image intensity; Ress et al. 2000) and also significantly smaller than the attention responses reported below.

To remove any systematic contribution of visual stimulation to the topographic maps, each segment had a $50 \%$ probability of containing a grating on each trial. Because these probabilities were independent of each other, on a given trial, the presence or absence of a target in one segment did not predict whether a target was present or absent in any other segment. Therefore there was no systematic visual stimulation, as a random pattern of target-present and target-absent segments appeared on each trial.

\section{Passive visual stimulation}

Functional MRI responses to passive visual stimulation were measured using a block alternation protocol. Subjects maintained fixation while viewing a stimulus in a peripheral annulus around the fixation point (same size annulus as in the attention-mapping experiments). The annulus alternated either every 9 or every $12 \mathrm{~s}$ between a checkerboard pattern $(100 \%$ contrast, either 2 or $3 \mathrm{cyc} / \mathrm{deg}, 4-\mathrm{Hz}$ contrast reversal) and a uniform gray field.

\section{fMRI data acquisition}

MR data were acquired using one of two 3-Tesla scanners located either at Stanford University or New York University (Stanford, General Electric Signa LX, Milwaukee, WI; New York University, Siemens Allegra, Erlangen, Germany), each equipped with a custom surface coil (Stanford, NMSC-002-TR-3GE transmit-receive coil, Nova Medical, Wakefield, MA; New York University, NM-011 trans- mit head-coil and NMSC-021 4-channel phased-array receive coil, also from Nova Medical). Scan parameters are summarized in Table 2. Slices were angled at approximately $45^{\circ}$ between axial and coronal to provide coverage of dorsal occipital and posterior parietal cortex. The surface coils were centered on dorsal occipital cortex to optimize the signal-to-noise ratio in posterior parietal and occipital cortex.

In addition to the functional images, a set of T1-weighted anatomical images was collected at the beginning of each scanning session using the same slice prescription used for the functional data. These in plane anatomical images were aligned to a high-resolution anatomical volume of each subject's brain using custom software (Nestares and Heeger 2000), so that the functional data across multiple scanning sessions from a given subject were coregistered to an accuracy of about $1 \mathrm{~mm}$.

High-resolution whole-brain T1-weighted anatomical images were acquired with a birdcage-style head coil on either a 3-Tesla Siemens Allegra scanner using a 3-dimensional (3-D) MPRAGE sequence (New York University) or on a 1.5-Tesla GE Signa LX scanner using a similar, inversion-recovery prepared 3-D SPGR sequence (Stanford). Voxel size was either $1 \times 1 \times 1 \mathrm{~mm}$ (New York University) or $0.9375 \times 0.9375 \times(1.2$ or 1.3$) \mathrm{mm}$ (Stanford)

\section{fMRI data analysis}

The first half-cycle of each fMRI scan (either 12 or $18 \mathrm{~s}$ ) was discarded to remove transient activity or artifact associated with the initiation of scanning. These included artifacts arising from incomplete magnetic saturation, the absence of steady-state hemodynamics, or differences in task performance during the first few trials of the scan. The remaining data were preprocessed by: 1) motion correction (see following text); 2) slice-timing correction to correct for the fact that the individual slices were not acquired simultaneously during each volume acquisition; 3) high-pass filtering the time series at each voxel to compensate for the slow drift that is typical in fMRI measurements (Smith et al. 1999); 4) dividing each voxel's time series by its mean intensity to convert the data from arbitrary image intensity units to percentage signal modulation and to compensate for the decrease in mean image intensity with distance from the receive coil; and 5) averaging across repeated clockwise and counterclockwise scans (see following text).

The average time-series data were analyzed by fitting a sinusoid (same period as that of the attention cycle) to the time series at each voxel and computing: 1 ) the correlation (technically called the coherence) between the time series and the corresponding best-fitting sinusoid and 2) the phase of the best-fitting sinusoid. The coherence quantified signal-to-noise (Engel et al. 1997), taking a value near 1 when the fMRI signal modulation at the period of the attention cycle was large relative to the noise (at the other frequency components) and a value near 0 when the signal modulation was small compared with the noise. The response phase measured the temporal delay of the fMRI signal relative to the beginning of the attention cycle and therefore corresponded to the polar-angle component of the topographic map.

TABLE 2. fMRI scan parameters

\begin{tabular}{lll}
\hline \hline \multicolumn{1}{c}{ Parameter } & \multicolumn{1}{c}{ Stanford } & \multicolumn{1}{c}{ New York University } \\
\hline Scanner & General Electric Signa LX & Siemens Allegra \\
fMRI acquisition & Spiral (Glover and Lai 1998; Glover 1999) & Echo planar imaging (EPI) \\
Echo time (TE) & $30 \mathrm{~ms}$ & $30 \mathrm{~ms}$ \\
Repetition time (TR) & $1.2 \mathrm{~s}$ & $1.2 \mathrm{~s}$ \\
Flip angle & $66 \mathrm{deg}$ & $75 \mathrm{deg}$ \\
\# of shots & 2 & 1 \\
Field of view & $220 \times 220 \mathrm{~mm}$ or 240 $\times 240 \mathrm{~mm}$ & $192 \times 192 \mathrm{~mm}$ or $220 \times 220 \mathrm{~mm}$ \\
In plane voxel size & $2.5 \times 2.5 \mathrm{~mm}$ or $2.73 \times 2.73 \mathrm{~mm}$ & 19 \\
\# of slices & 20 & $3 \mathrm{~mm}$ \\
Slice thickness & $3 \mathrm{~mm}$ & $3.44 \mathrm{~mm}$ \\
\hline
\end{tabular}


The statistical significance of a given coherence value can be computed using the following formula (Sokal and Rohlf 1995)

$$
\mathrm{t}=\mathrm{r} \sqrt{\frac{\mathrm{n}-2}{1-\mathrm{r}^{2}}}
$$

where $t$ equals the value of the $t$-statistic, $r$ equals the coherence value, and $n$ equals the number of data points, in this case the number of time points in a single run. For a given coherence value, the $t$-statistic can be easily converted into a $P$ value to indicate the level of statistical significance. The null hypothesis is that there is no significant signal modulation at the period corresponding to the attention cycle compared with noise at other frequencies. This analysis assumes that the noise is uncorrelated, an assumption known to be false for fMRI time series. In fact, temporal autocorrelation in blood oxygenated leveldependent (BOLD) fMRI noise has been effectively modeled with a $1 /$ frequency function (Zarahn et al. 1997). Therefore the reported $P$ values should be considered to be rough estimates of the levels of statistical significance of the associated coherence values.

To minimize head movements, subjects were stabilized on a bite bar. The time series of fMRI data from each scan were inspected for large head movements, which were evident as large steps or impulses in the time series, using software developed in our lab. Scans showing evidence of such large head movements were discarded from further analysis. Small residual head movements were corrected using a 3-D image registration algorithm (MCFLIRT; Jenkinson et al. 2002). This motion correction also compensated for small image shifts in the New York University data that were caused by gradual heating of gradient coils, which in turn heated the fixed shims and slightly changed the magnetic field strength.

The data from the $\mathrm{CW}$ and $\mathrm{CCW}$ scans were combined to compensate for the hemodynamic delay. The time series from the two types of scans were temporally aligned by advancing both by a fixed amount of time (corresponding approximately to the hemodynamic delay) and then time-reversing the $\mathrm{CCW}$ scans. The $\mathrm{CW}$ and time-reversed $\mathrm{CCW}$ time series were then averaged to eliminate the hemodynamic delay. Because this procedure was performed for each voxel independently, it compensated correctly for the hemodynamic delays even if they were different in different cortical regions. The precise choice of temporal shift was not critical because it did not affect the resulting phase of the averaged CW and CCW scans (although it did have an effect on the amplitude of the response modulations). The time shift was therefore chosen to maximize the coherence, on average (across scans and across voxels in and near the IPS), separately for each subject.

\section{Flat maps and data visualization}

To visualize the results of the topography experiments, we rendered the response phases on a computationally flattened representation ("flat map") of cortical gray-matter voxels within relevant regions of each subject's brain using custom software developed in our lab (Larsson 2001) and in collaboration with colleagues at Stanford (Teo et al. 1997; Wandell et al. 2000).

\section{Defining cortical areas}

Early visual areas (V1, V2, V3, V3A, V3B, and V7) were defined using well-established methods that have been extensively described (DeYoe et al. 1996; Engel et al. 1994, 1997; Sereno et al. 1995). Briefly, we presented rotating and expanding (or contracting) stimuli that evoked traveling waves of activity in retinotopic visual areas. The visual areas were then restricted in subsequent sessions based on responses to the passive visual stimulation scans (see above), in which a contrast-reversing checkerboard annulus alternated with a neutral gray screen. The checkerboard annulus was the same size and shape as the annulus used for attention mapping, so the restricted visual areas corresponded retinotopically to the attended annulus.

The topography of visual responses that we observed in dorsal extrastriate cortex most closely matched the organization described in Press et al. (2001) and reviewed in Wandell et al. (2005). Specifically, cortical areas V3A, V3B, and V7 each contained a full contralateral hemifield representation. Other studies described the boundaries of $\mathrm{V} 3 \mathrm{~A}$ as encompassing the area we refer to as V3B (Tootell and Hadjikjani 2001; Tootell et al. 1997, 1998) or reported that V3B contained only a lower contralateral visual quadrant representation (Dumoulin et al. 2003; Smith et al. 1998).

The responses in the attention-mapping task clearly indicated at least two topographically organized areas within the IPS. The general pattern of the topography was consistent across our sample of 10 hemispheres from 5 subjects based on: the location of each contralat-

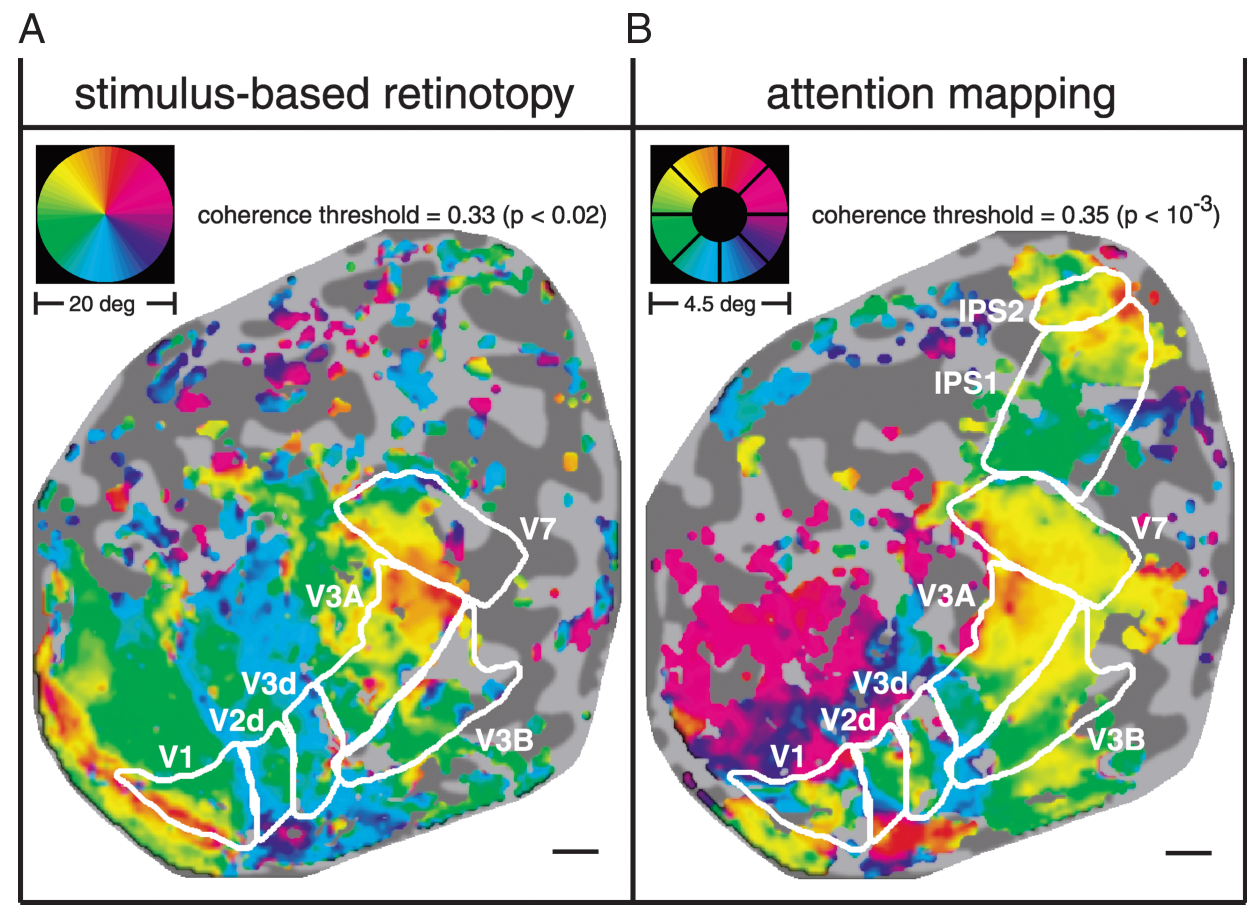

FIG. 2. Correspondence between stimulusbased retinotopic and attention-related maps. $A$ : angular component of retinotopic maps, measured using conventional visual stimulation (see RESULTS for details). Gray scale flattened representation of cortical anatomy corresponding to occipital and parietal cortex (subject MAS, right hemisphere). Dark shading corresponds to sulci and lighter shading to gyri. Color: fMRI response phase. Color wheel inset: corresponding angular position in the visual field. Colors are displayed for all gray matter locations that exceeded the indicated coherence threshold. For each panel, the $P$ value corresponding to the coherence threshold (see METHODS) is also shown. Scale bar, about $1 \mathrm{~cm}$. $B$ : attention-related maps (same format as $A$ ). Indicated visual areas V1 through V7 were defined using stimulus-based retinotopic mapping, i.e., copied from $A$. In addition, there are two additional cortical maps, labeled IPS1 and IPS2, that are not evident in the stimulus-evoked retinotopic maps. 
eral visual field map with respect to the gross anatomy (mainly the transverse occipital sulcus and intraparietal sulcus), the size of each map, the distances between the maps and their location relative to one another, and the visual field orientations of the topographic maps. The coherence threshold used for visualizing the maps varied from subject to subject (range of 0.30 to 0.59 ) and was mainly a function of the number of scanning sessions per subject. Boundaries between proposed cortical areas were drawn by hand on the flat maps, guided by the spatial organization of response phases and coherence values.

Talairach coordinates were determined in each subject for cortical areas V3A, V3B, V7, IPS1, and IPS2 using custom software (mrVISTA, available for free download at http://white.stanford.edu/ software/). Talairach coordinates reported in Sereno et al. (2001) were defined with respect to the MNI (Montreal Neurological Institute) template brain (not the Talairach brain). To allow a direct comparison of these data and our Talairach coordinates, the coordinates from Sereno et al. (2001) were transformed into Talairach space using MATLAB code written by M. Brett and freely available at http:// www.mrc-cbu.cam.ac.uk/Imaging/Common/mnispace.shtml

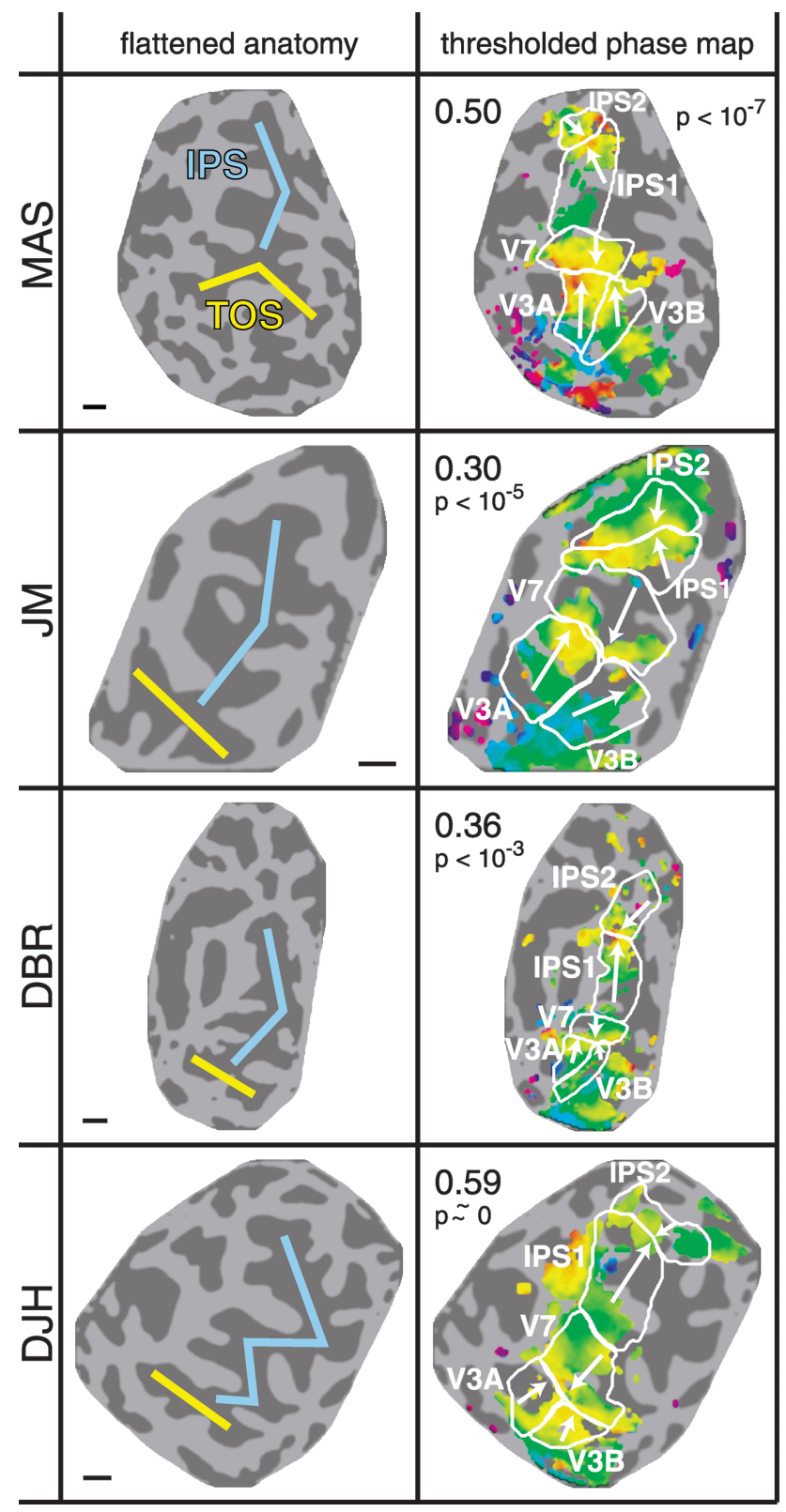

\section{Computing visual field orientation}

There are two diagnostic features of retinotopic phase maps in early visual cortex: 1) the response phases progress across each visual area (indicating a topographic organization of the visual field on the cortical surface) and 2) there are phase reversals located at boundaries between adjacent visual areas that share a common visual field meridian representation (DeYoe et al. 1996; Engel et al. 1997; Sereno et al. 1995). The adjacent maps are mirror images of each other, so the phase progressions are in opposite directions along the cortical surface, resulting in a phase reversal at their shared boundary. To quantify the reversals in the topographic maps, we computed the visual field orientation for each proposed cortical area. The local visual field orientation at each voxel was determined by comparing its response phase to the phases of each of its 8 neighboring voxels on the flat map. In particular, the local horizontal component of visual field orientation was determined by: 1) computing the difference between the sine of the response phase of the central voxel and the sine of the phase of each of its neighboring voxels and 2) computing the difference between the resulting values from the 3 voxels on the right minus
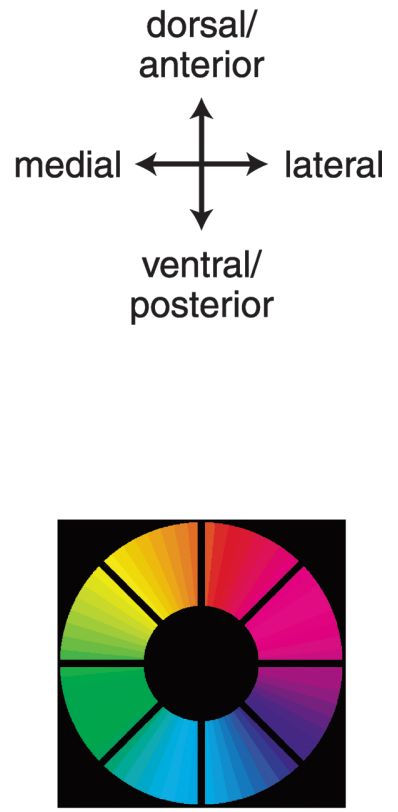

FIG. 3. Right hemisphere attention-related maps in parietal cortex for all 4 subjects. Left column: flattened representations of posterior parietal cortex. Cyan curve, intraparietal sulcus (IPS); yellow curve, transverse occipital sulcus (TOS). Right column: fMRI response phases with cortical area boundaries superimposed (same format as Fig. 2). Indicated areas V3A, $\mathrm{V} 3 \mathrm{~B}$, and $\mathrm{V} 7$ were defined using stimulus-based retinotopic mapping. Proposed IPS1 and IPS2 areas were defined based on the attention-mapping results. Coherence thresholds were chosen separately for each hemisphere and are displayed next to the thresholded phase maps. For each map, the $P$ value corresponding to the chosen coherence threshold is also shown. Arrows indicate visual field orientation, pointing toward the upper vertical meridian and away from the lower vertical meridian representation. Scale bars, about $1 \mathrm{~cm}$. 
the values from the 3 voxels on the left. The local vertical component of the visual field orientation was determined analogously. The visual field orientation of an entire cortical area was computed as the vector average of the local orientations at each voxel. Although the phase maps shown in Figs. 2-4 have been thresholded to show the voxels that were best correlated with the attention task, all quantitative analyses in this paper, including the visual field orientation, were computed using all voxels within each proposed cortical area.

\section{Eye position recordings}

Eye position was measured within the MR scanner using an infrared videographic camera (Applied Sciences Laboratories, Bedford, MA). The acquisition rate was $60 \mathrm{~Hz}$. Each scan contained 6 cycles of attention mapping with continuous fixation followed by an additional cycle in which subjects performed the same task but made overt eye movements to the attended segment. The eye position traces during these eye movement trials were used to spatially calibrate the eye position data and to transform them into units of degrees of visual angle.

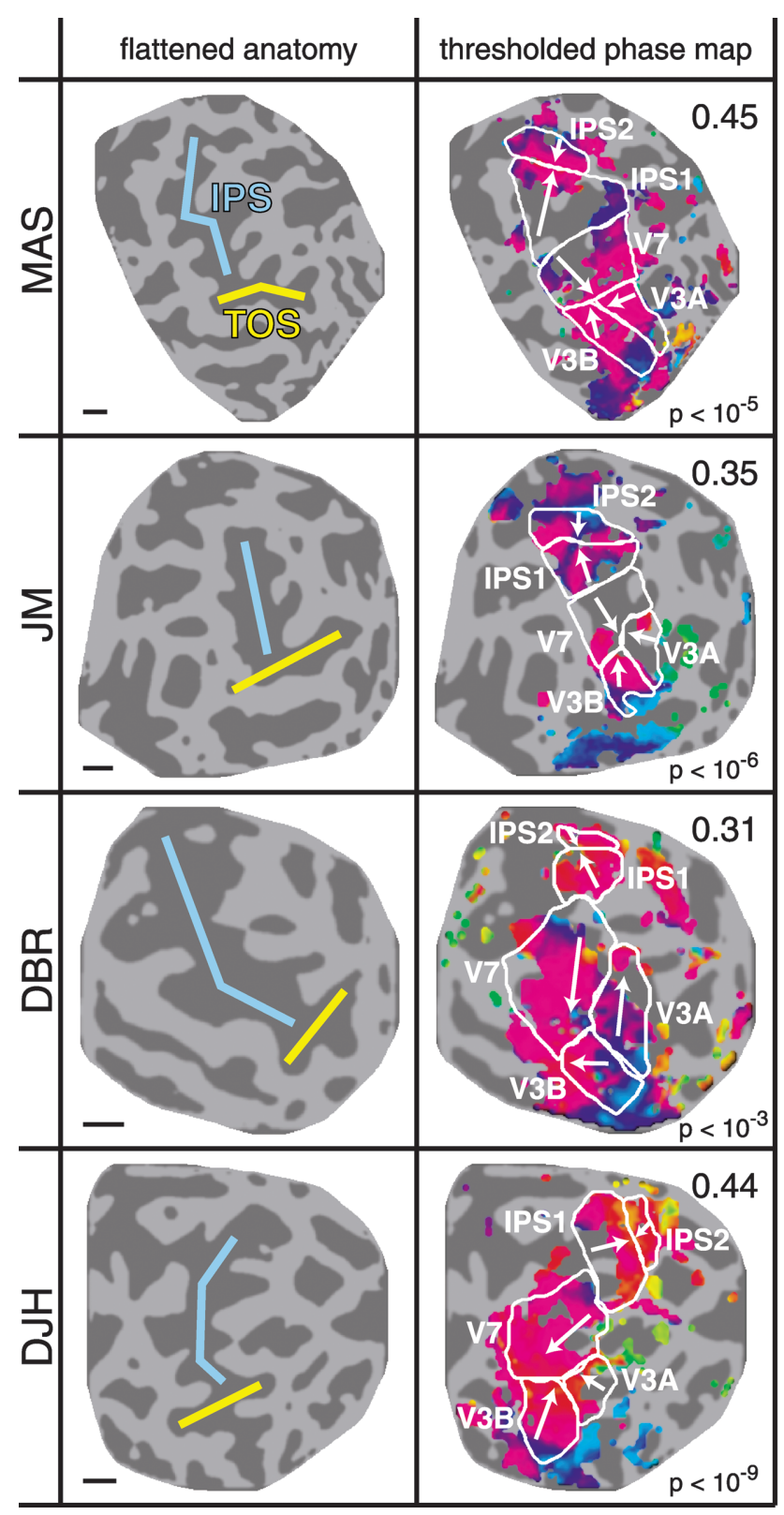

Eye movement data were analyzed with custom software to measure fixation stability. Data were calibrated and then warped to visual space using a 3rd-order polynomial-fitting algorithm. Saccades, blinks, and artifacts were identified with automated procedures but checked manually for accuracy. Fixation was verified by: 1) inspecting the data manually for saccades to the cued target locations, 2) measuring the SD of eye position during fixation epochs, and 3) measuring the amplitude of modulation of eye position in phase with the cued location. Using these methods, we monitored fixation with an accuracy of about $0.5^{\circ}$ (see RESULTS).

\section{R E S U L T S}

To identify posterior parietal regions containing topographic maps of visual spatial attention, we used a task in which the focus of attention systematically traversed the visual field (Fig. 1). Subjects performed a visual detection task in which the target was a low-contrast grating presented at the threshold of visibility as determined separately for each subject (Table 1).
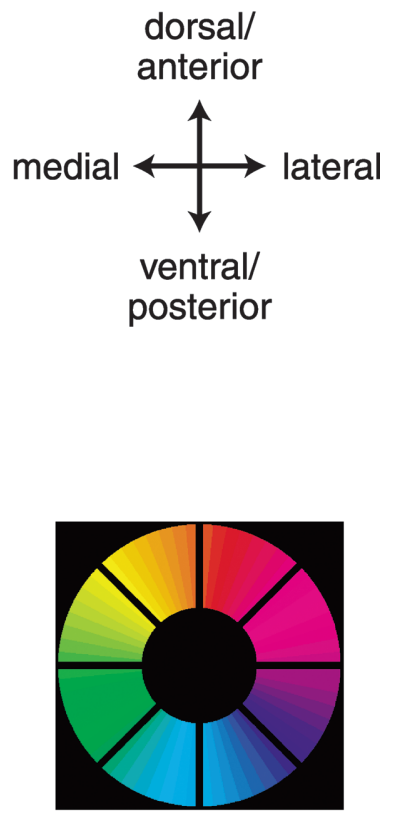

FIG. 4. Left hemisphere attention-related maps in parietal cortex for all 4 subjects (same format as Fig. 3). 
The responses in the flat maps (Figs. 2-5) should be read as follows. Each panel displays a flat map of one hemisphere. The superimposed colors represent the fMRI response phase that corresponds to angular position in the visual field (see METHODS). The responses are lateralized such that one set of colors (green, yellow) appear in the right hemispheres whereas a different set of colors (blue, magenta) appear in the left hemispheres. Responses to the upper and lower vertical meridia (orange and cyan, respectively) can be found in both hemispheres. The arrows indicate the smooth progression in topography from the lower vertical to the horizontal and then to the upper vertical meridian as one moves across the cortical surface (Figs. 3 and 4). There are clear phase reversals (reversals in the orientation of the topographic maps), indicated by arrows in adjacent cortical areas pointing in opposite directions. It is known that such phase reversals, in early visual cortex, correspond to boundaries between cortical areas (DeYoe et al. 1996; Engel et al. 1997; Sereno et al. 1995).

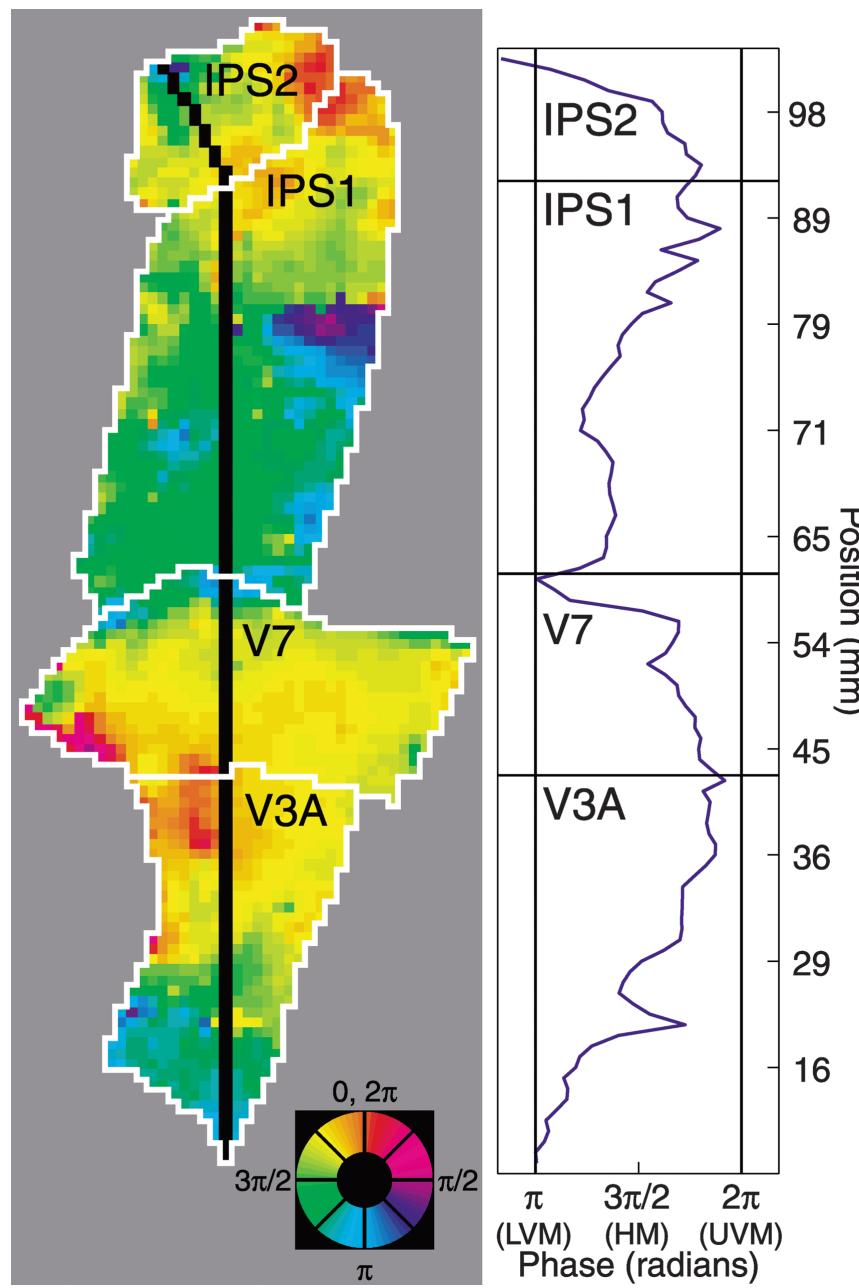

FIG. 5. Phase progressions within cortical areas and phase reversals between adjacent areas. Left: unthresholded, unsmoothed attention-related maps (subject MAS, right hemisphere). Black line indicates linear region of interest (ROI). Color wheel: angular position in the visual field. Right: response phase as a function of position along the black line. In each cortical area, the phases span the entire contralateral visual field. Vertical meridian representations occur at the boundaries between cortical areas and are associated with reversals of phase. Distances were measured in the folded cortical manifold (the boundary between the cortical white and gray matter) to avoid spatial distortions inherent in the flattening process (Dougherty et al. 2003).
Colors are displayed for all gray-matter voxels that exceeded the indicated coherence threshold (see METHODS). Thus there are regions that appear noisy and there are "dropouts" (uncolored patches) where the coherence did not exceed threshold. The coherence thresholds were chosen separately for each hemisphere and are displayed next to the thresholded phase maps. Coherence thresholds were chosen by hand to best reveal the topographic organization in posterior parietal cortex. The maps appeared noisier for lower thresholds and there were more dropouts for higher thresholds, as expected for an arbitrary statistical threshold. A post hoc analysis demonstrated that the chosen coherence thresholds were correlated with the number of scanning sessions conducted for each subject.

We found several regions in the dorsal extrastriate cortex and PPC that consistently exhibited a topographic organization of responses during performance of the attention-mapping task (Fig. 2B, example hemisphere; Figs. 3 and 4, all hemispheres). Three of these regions corresponded to visual areas $\mathrm{V} 3 \mathrm{~A}, \mathrm{~V} 3 \mathrm{~B}$, and V7, as defined by conventional stimulus-based retinotopic mapping techniques (see following text). The other regions (labeled IPS1 and IPS2 in the figures) were identified in both hemispheres of all 4 subjects (Figs. 3 and 4). The proposed IPS1 region shared a boundary with dorsal/ anterior V7 corresponding to the lower-vertical-meridian (LVM) representation. The phases progressed dorsally and anteriorly until the upper-vertical-meridian (UVM) representation was reached, which corresponded to the proposed boundary between IPS1 and IPS2. There was a phase reversal at this boundary, and the phases progressed anteriorly and dorsally through the proposed IPS2 region from the UVM to the LVM. In some hemispheres, there was an additional phase reversal at the dorsal-anterior boundary of IPS2, but the organization of the response phases beyond IPS2 was not consistent enough across hemispheres to draw clear conclusions about the possible existence of distinct topography beyond IPS2. The variability in this region is probably explained by the fact that the receive coil was farther from these areas, so signal-to-noise ratios in the data were lower.

The reversals in the topographic maps were verified using an objective measure of visual field orientation. The direction of visual field orientation (vectors in Figs. 3 and 4) was quantified for each cortical area by comparing the fMRI response phase of each voxel with that of the 8 neighboring voxels on the flat maps (see METHODS). The vectors pointed in nearly opposite directions in pairs of adjacent maps, indicating a sequence of phase reversals between the topographic maps in (V3A and B), V7, IPS1, and IPS2. It should be noted that the visual field orientation measurement depended on the proposed boundaries between the cortical areas and was thus largely redundant with the underlying phase maps. It did, however, provide a single metric that summarized a complex spatial pattern of response phases and allowed for verification of reversals in visual field layout on the cortical surface.

The dorsal/anterior boundary of V7 (and therefore the proposed V7/IPS1 border) was defined using stimulus-based retinotopic mapping, and the location of the border was typically marked by a phase reversal in the attention-mapping experiments. The fact that these two methods corroborate each other allowed the V7/ IPS1 boundary to be established with a high degree of confidence. Additionally, the phase reversal at the proposed IPS1-IPS2 boundary was evident in the phase maps for all 8 hemispheres and was confirmed by the nearly $180^{\circ}$ difference in the visual field orientations for IPS1 and IPS2 in each hemisphere. 
The phase progressions within areas and the phase reversals between areas can be demonstrated by plotting the response phase versus position along a line that spans multiple areas. The example flat map in Fig. 5 displays every voxel (i.e., the coherence threshold was equal to zero), and no spatial smoothing was performed. For example, cortical areas V3A and V7 each contained a complete contralateral hemifield representation (response phases included nearly all values between $\pi$ and $2 \pi$ ), and the response phases were topographically organized within each cortical area (the response phases progressed smoothly). In addition, the visual field representations in these adjacent cortical areas were mirror images of each other. Similar results were evident dorsal/anterior to V7, suggesting the presence of a pair of contralateral hemifield representations within the IPS (the proposed IPS1 and IPS2 areas).

To characterize the laterality of these cortical areas, we plotted the distribution of fMRI response amplitudes and phases in each hemisphere (Fig. 6). Overall, the visual field representations of the voxels in IPS1 and IPS2 corresponded to the contralateral visual field. The plots in Fig. 6 contain all the gray-matter voxels within IPS1 and IPS2, including those that had coherence values below the threshold for visualization in Figs. 2-4. For left IPS1, the average percentage of voxels that represented the right contralateral hemifield was $83 \%(n=4$ hemispheres), and for right IPS1, the average was $85 \%$. The corresponding mean values for IPS 2 were $90 \%$ in the left hemisphere and $90 \%$ in the right hemisphere.

In general, the response phases in PPC were overrepresented near the contralateral horizontal meridian representation and underrepresented near the vertical meridian. A number of factors could contribute to this anisotropic distribution of response phases. 1) The BOLD signal combines activity from many neurons in a single voxel, thereby effectively averaging many response fields. This "pooled response field" could have a large apparent size, even though the constituent neuronal response fields would be much smaller. It is not possible for the center of this pooled response field to be located near the vertical meridian unless some of the constituent response fields were in the ipsilateral hemisphere (Press et al. 2001). 2) Limitations on temporal and/or spatial resolution in the phase maps would have resulted in a relative underrepresentation of phases near the vertical meridian. 3) Finally, it is possible that this anisotropy in visual topography is a general feature of the primate visual system. The distribution of retinal ganglion cells reflects an overrepresentation of neurons along the horizontal meridian in several primate species, including human (Stone and Johnston 1981), and the same anisotropy has been observed with fMRI in the human lateral geniculate nucleus (Schneider et al. 2004) and visual cortex (Janik et al. 2003). Regardless of the cause of the anisotropy, the underrepresentation of phases near the vertical meridian limited the precision with which we could define the proposed IPS1-IPS2 border, because this border represented the upper vertical meridian.

The sizes and locations of the proposed IPS1 and IPS2 regions were comparable across subjects and hemispheres. The locations of IPS1 and IPS2 (as defined using the Talairach coordinate system) were approximately the same for both hemispheres in all 4 subjects (Table 3). To avoid spatial distortions introduced by the flattening procedure, cortical area sizes were measured in the 3-D cortical manifold (the boundary between the cortical white and gray matter) using custom software (Dougherty et al. 2003). The size of IPS 1 varied by a factor of 3.4 across the 4 subjects in our study, and the size of IPS2 varied by a factor of 1.4. This

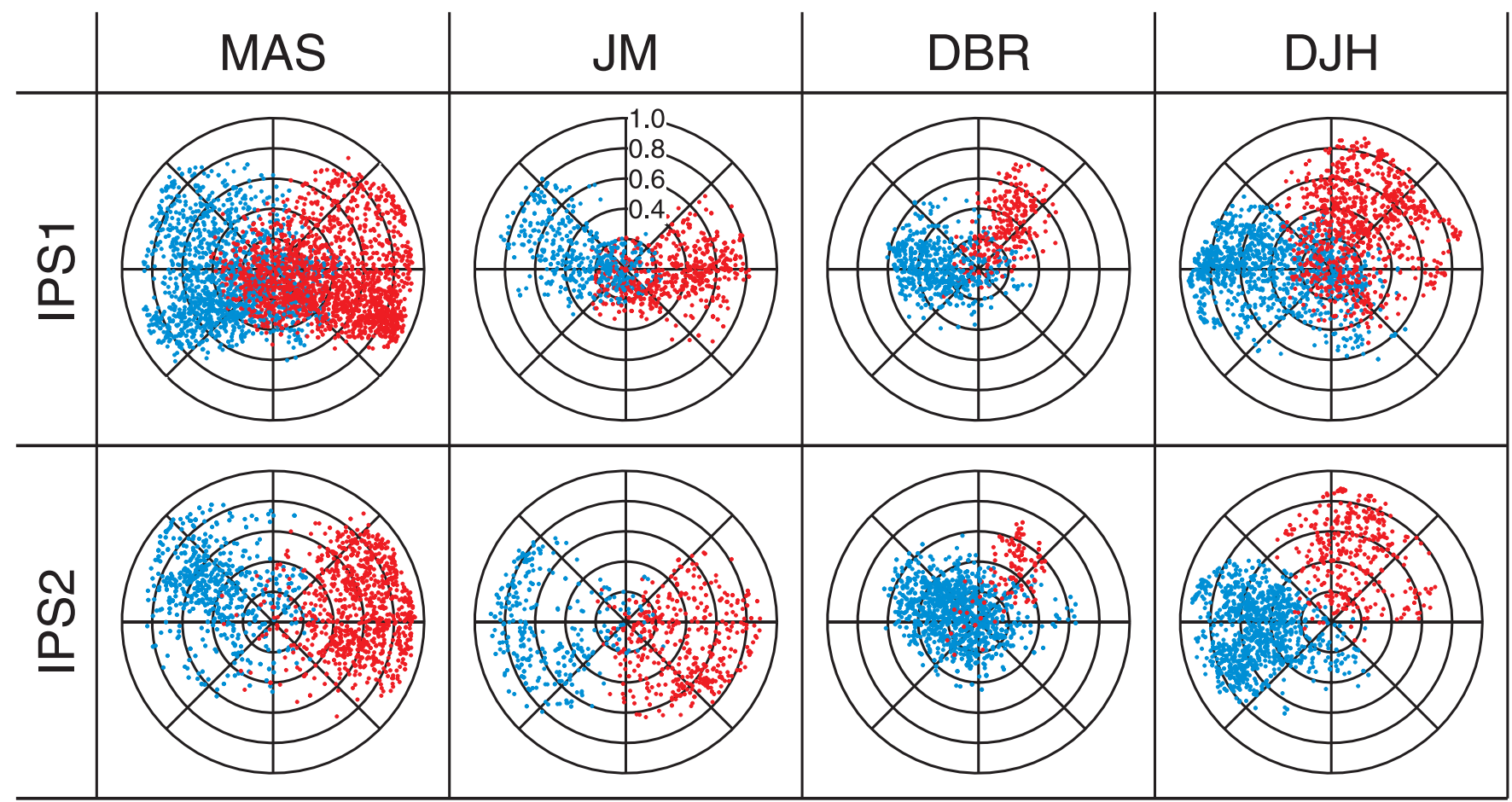

FIG. 6. Attention-related responses in IPS1 and IPS2 represent the contralateral visual hemifield. Each point corresponds to a single gray matter voxel. Red points are from left hemispheres and blue points are from right hemispheres. Angular position represents visual field location within the attended annulus, and radial position is the coherence coefficient. Although the maps in Fig. 3 and Fig. 4 are thresholded and display only those voxels most correlated with the attention cycle, the plots in this figure include every voxel within each of the cortical areas. 
TABLE 3. Talairach coordinates for parietal cortical areas

\begin{tabular}{lcccc}
\hline \hline Subject & V3A & V3B & V7 & IPS1 \\
\hline MAS & $\pm 22,-89,19$ & $\pm 28,-89,12$ & $\pm 24,-81,24$ & $\pm 25,-76,42$ \\
JM & $\pm 25,-84,13$ & $\pm 30,-86,8$ & $\pm 25,-81,24$ & $\pm 24,-78,37$ \\
DBR & $\pm 19,-86,19$ & $\pm 24,-90,17$ & $\pm 28,-81,22$ & $\pm 23,-73,31$ \\
DJH & $\pm 22,-91,25$ & $\pm 29,-91,20$ & $\pm 27,-82,30$ & $\pm 22,-77,46$ \\
Mean & $\pm 22,-87,19$ & $\pm 28,-89,14$ & $\pm 26,-81,25$ & $\pm 2,-76,39$ \\
SD & $5,3,6$ & $6,5,5$ & $5,2,4$ & $5,5,6$ \\
\hline
\end{tabular}

Cortical areas V3A, V3B, and V7 were defined using stimulus-based retinotopic mapping and were restricted based on visual responses to a contrast-reversing checkerboard stimulus that had the same shape and size as the annulus used for attention mapping. IPS1 and IPS2 boundaries were defined based only on the responses during the attention-mapping task.

variability is comparable to previously reported intersubject variability in the sizes of early visual cortical areas. Specifically, V1, $\mathrm{V} 2$, and V3 have been reported to vary by a factor of about 2.5 across subjects (Dougherty et al. 2003).

The boundaries of visual areas V1, V2d (the dorsal half of V2), V3d (the dorsal half of V3), V3A, V3B, and V7 were defined using standard stimulus-based retinotopic mapping methods (see Fig. 2 for an example). These cortical areas were further restricted based on retinotopic responses to a highcontrast checkerboard annulus that was the same size and shape as the annulus used in the attention-mapping scans (see METHODS). Thus the V1-V7 boundaries indicated in the figures throughout this paper should be interpreted as representing the portion of each cortical area ranging from about 0.75 to $2.25^{\circ}$ of eccentricity.

Maps of attention-related activity in areas V1, V2d, V3d, V3A, V3B, and V7 corresponded well with the stimulus-based retinotopic maps (compare Fig. 2, $A$ and $B$ ). This was evident for the example hemisphere shown in Fig. 2, and it was generally the case in all hemispheres. Correspondence between stimulus-based retinotopy and attention topography has been previously reported in visual cortex (Brefczynski and DeYoe 1999; Kastner et al. 1999; McMains and Somers 2004; Sasaki et al. 2001; Tootell et al. 1998). In the present study, this correspondence was clearly evident in all visual cortical areas from V1 through V7. In addition, the attention-related maps exhibited strikingly precise topography and, for some cortical areas, they were indistinguishable from the maps obtained using stimulus-based retinotopic mapping.

The stimulus-based retinotopic and attention-related maps were very different from one another in portions of V1, V2d, and V3d corresponding to peripheral eccentricities. This was attributed to the fact that large $\left(20^{\circ}\right.$ diameter) stimuli were used to generate the stimulus-based retinotopic maps, whereas the attention-mapping task used a small annulus ( $4.5^{\circ}$ diameter). Thus in Fig. $2 A$ (stimulus-based retinotopy), the retinotopic organization in V1, V2d, and V3d extends leftward and upward from the central representation (where the cortical areas are indicated) to the peripheral representation of the visual field. In the attentionrelated map (Fig. 2B), the visual field representations of the peripheral eccentricities contain blue, red, and magenta voxels, nearly $180^{\circ}$ out of phase with the responses at the corresponding cued locations in the central visual field. This out-of-phase activity may reflect an inhibitory penumbra in the "spotlight" of attention, that is, a decrease in the responses in visual field representations surrounding the attended region (Müller and Kleinschmidt 2004; Smith et al. 2000; Tootell et al. 1998).
Although PPC exhibited clear topographic organization in our attention-mapping protocol, the proposed IPS1 and IPS2 areas have not been described in previous stimulus-based retinotopic mapping studies, suggesting that their responses during passive viewing may be small relative to responses in early visual cortical areas. To assess this directly, we measured the fMRI response amplitudes during passive viewing of a high-contrast visual stimulus and during performance of the attention-mapping task, which used very low contrast stimuli. We observed a gradient of amplitude values for responses to passive visual stimulation, with early visual areas exhibiting the largest values and PPC areas having the smallest values (Fig. 7A). However, further experiments are needed to determine the generality of this result because these areas differ greatly in their receptive field sizes and stimulus selectivity properties. It should be noted that the responses to passive viewing of visual stimuli may not represent purely sensory responses. Although subjects were maintaining fixation throughout the passive viewing scans, they may have allocated attention to the high-contrast visual stimulus. Thus the responses during the passive viewing scans may reflect a combination of sensory and attention signals. In contrast to the differences in response amplitude across cortical areas in response to passive visual stimulation, the responses to the attention-mapping task were nearly equal in early visual, dorsal extrastriate, and PPC areas (Fig. 7B).

Although subjects were instructed to maintain fixation throughout the attention-mapping task, it is possible that occasional eye movements toward the attended segment generated activity correlated with the attention task. This would have confounded attention-related and eye movement-related activity in our measurements. To address this issue, we measured

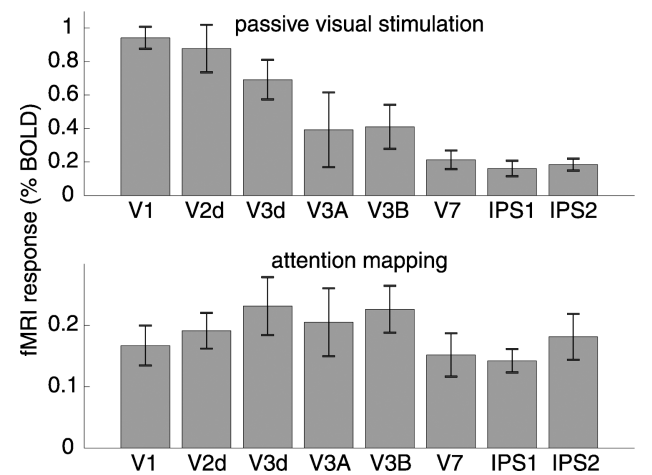

FIG. 7. Responses to passive visual stimulation and attention. A: fMRI response amplitudes to passive visual stimulation with a high-contrast visual stimulus. $B$ : fMRI response amplitudes during performance of the attentionmapping task (with very low-contrast visual stimuli). For a given cortical area, the response amplitudes were computed for each subject and then averaged across subjects $(n=4)$. Error bars: SE. 
subjects' eye position during performance of the attentionmapping task in the scanner (Fig. 8). Eye position was recorded during performance of 6 cycles of covert attention. Immediately after the end of the 6th cycle, subjects performed an additional cycle that was exactly the same as the attentionmapping task, but this time the fixation marker moved to the cued location, indicating to the subjects that they should move their eyes (as well as their attention) to that location, while still performing the task as before. This additional cycle simulated the pattern of eye movements expected if subjects did not
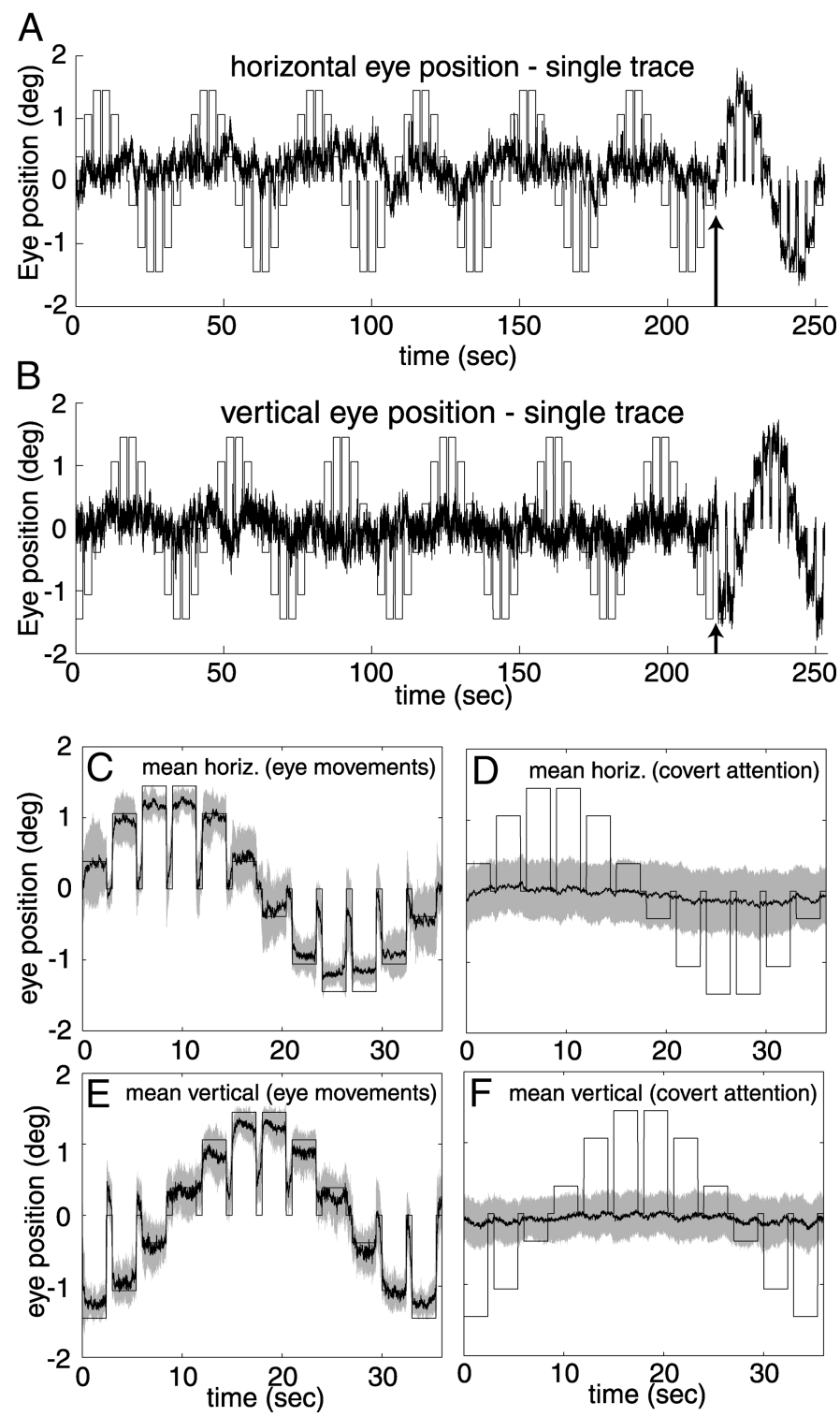

FIG. 8. Eye position during attention mapping. A: single example of horizontal eye position recorded in the MR scanner from subject DJH. Black trace, measured eye position. Bars, expected horizontal eye position if the subject made eye movements to the attended segment on every trial. At the time point indicated by the arrow, the screen was blanked for $1.2 \mathrm{~s}$, instructing the subject to make saccades to the attended segment on the subsequent trials. $B$ : vertical eye position from the same example shown in $A$. $C$ : mean horizontal eye position during performance of the eye movement trials for subject DJH. Shaded region, \pm 1 SD. $D$ : mean horizontal eye position during performance of the covert attention task. $E$ : mean vertical eye position during eye movement trials. $F$ : mean vertical eye position during covert attention trials. Eye position was very weakly correlated with the cued location when the subject was instructed to maintain fixation. maintain fixation but rather made a saccade to the attended segment on each trial. It also served as a positive control for the eye movement measurements.

There was a slight bias in eye position toward the cued target locations during the attention-mapping scans (Fig. 8). Figure 8, $A$ and $B$ shows typical examples of single traces of eye position during the performance of 6 covert attention cycles followed by one eye movement cycle. Large changes in eye position were evident during the last cycle when subjects were instructed to make overt eye movements to the cued location. Otherwise, there was no evidence that subjects shifted their gaze away from the central fixation point to the cued target location during this scan, nor during any of the other scans. Figure $8, C-F$ plot average eye positions from a total of 6 scanning sessions in 2 subjects. Visual inspection of these graphs shows that the eye movement scans are highly correlated with the expected pattern of eye movements when subjects were instructed to make saccades to the attended location on each trial. For covert attention, on the other hand, the averaged eye traces are only slightly biased toward cued locations, indicating that subjects effectively maintained fixation while performing the task in the periphery.

To quantify the relationship between eye position and target location, we computed a pair of correlation coefficients for each scan (Fig. 9). When subjects were cued to move their eyes, the correlation between eye position and cued location was very high (Fig. 9A). The mean correlation coefficient was 0.87 , significantly different from zero $(P \approx 0$, one-tailed $t$-test). During covert attention, this correlation was much weaker (Fig. $9 B$, mean correlation coefficient of 0.13 ), but it was still significantly different from zero $(P \approx 0$, one-tailed $t$-test $)$. Thus there is at least a formal possibility that some of the topographic activity in PPC resulted from imperfect eye fixation.

To rule out this possibility, we performed a median split based on the correlation coefficients during covert attention. fMRI responses from scans with low correlation coefficients were pooled and displayed in the form of a phase map on flattened PPC (Fig. 9C). The identical analysis was performed on the scans that had the highest correlation coefficients and presumably the most eye movement-related activity (Fig. 9D). The two maps were extremely similar, and no systematic differences were evident. This result demonstrates that eye movements had little or no effect on the responses in parietal topographic maps during attention mapping.

\section{I S C U S S I O N}

Through the use of a visual field mapping technique that minimized sensory and eye movement signals, we have discovered that a portion of human PPC within the IPS contains topographically organized representations of the contralateral visual field. In addition, the pattern of phase progressions and phase reversals strongly suggests that the IPS topography can be subdivided into at least two full contralateral hemifield representations, which we have tentatively labeled IPS1 and IPS2 (Figs. 3 and 4). Several features of IPS1 and IPS2 were consistent across the hemispheres in our sample. 1) IPS1 was immediately adjacent to V7 and was located in the medial bank of the IPS. 2) IPS2 shared a boundary with IPS1 and was also located along the IPS. 3) Both areas contained contralateral visual field representations. 4) The boundaries between V7 and 



FIG. 9. Small bias in fixation toward the cued locations does not account for the observed topographic organization obtained with attention mapping. A: histogram of the correlation between the measured eye positions and the cued locations for blocks of trials in which subjects moved their eyes to the cued locations. Each event in the histogram corresponds to eye position data from a single scan (about 5 min), derived from 6 scanning sessions (2 for subject MAS and 4 for subject DJH) during which eye movements were recorded. $B$ : histogram of correlation coefficients from covert attention trials. Correlation coefficients are small but significantly greater than zero. $C$ : maps derived during covert attention from scans with low $(<0.122$ in panel $B)$ correlation coefficients (subject MAS, left hemisphere, same format as Fig. 2). $D$ : maps derived again during covert attention but from scans with high $(>0.122$ in panel $B)$ correlation coefficients. Maps in $C$ and $D$ are virtually identical, indicating that eye movements were very unlikely to substantially contribute to the observed topographic pattern of activity. Scale bar, $1 \mathrm{~cm}$.

IPS1 and between IPS1 and IPS2 were associated with phase reversals in the topographic maps. 5) The locations of IPS1 and IPS2 (in Talairach space) were similar for both hemispheres in all 4 subjects. Despite the consistency of IPS1 and IPS2 features in our sample, the quality of the attention-related maps was lower than that obtained in early visual areas using stimulus-based retinotopic mapping. This could be explained by a number of factors, including 1) large response fields in individual neurons in IPS1 and IPS2, 2) less precise topography in IPS1 and IPS2 than in retinotopic early visual areas, 3) the use of stimuli and/or tasks that were not optimal for maximizing responses in IPS1 and IPS2, and 4) the allocation of attention to a particular portion of the visual field being less precise than stimulus-evoked responses (Intriligator and Cavanaugh 2001).

Although the topography in PPC was observed to be consistent across subjects and hemispheres, there is some ambiguity as to the interpretation of these results. The data support the conclusion that there are two areas (IPS1 and IPS2), each containing a single contralateral hemifield representation. However, the possibility remains that future investigations of these areas will reveal multiple visual field representations that will require these areas to be further subdivided. One alternative interpretation (and the evidence against it) is presented in the companion paper (Schluppeck et al. 2005). The designations IPS1 and IPS2 should therefore be considered to be tentative. This choice of nomenclature is neutral with respect to the possible functions of these areas as well as their possible homologies with parietal cortical areas in the macaque.

\section{Functional specialization of IPS1 and IPS2}

Our results are consistent with an interpretation in which IPS1 and IPS2 are functionally specialized for controlling spatial attention. Although the IPS1 and IPS2 responses could also be interpreted as motor intention signals, the analysis of eye movements during the attention-mapping task (Fig. 9) argues against a significant contribution of overt eye movements to the responses we have measured.

Further experiments will be required to determine exactly which functional components of attention (or covert intention) are encoded by IPS1 and IPS2. Of particular interest is to characterize the role(s) of these cortical areas in shifting and/or maintaining attention, and in endogenous and/or exogenous attention. Voluntary shifts in attention are believed to be mediated by transient responses immediately after the presentation of an attention-directing cue (Posner et al. 1984; Yantis et al. 2002), whereas voluntary, or endogenous, maintenance of attention is believed to be mediated by sustained activity during a delay period after the cue (Bisley and Goldberg 2003; Corbetta et al. 2000; Goldberg et al. 2002; Hopfinger et al. 2000; Kusunoki et al. 2000; Shulman et al. 1999). In contrast, shifts in exogenous attention occur when attention is involuntarily reoriented in response to a salient stimulus. The neural substrates of endogenous and exogenous attention have been shown to be partially distinct from one another (Corbetta et al. 2000). In the present study, subjects performed a target detection task in which the attentional cues were auditory and the visual stimuli were presented at the threshold of detection. This task thus required endogenous attention and minimized the contribution of exogenous attention. This implicates IPS1 and IPS2 as having roles in endogenous attention but does not rule out the possibility that they are also active during exogenous attention.

Other experiments in our lab have demonstrated that IPS1 and IPS2 exhibit sustained responses during periods of maintained attention in the absence of sensory stimulation, and this sustained delay-period activity persists for as long as subjects maintain attention (at least as long as $16 \mathrm{~s}$, the longest period tested) (Silver et al. 2003). This does not, however, exclude the possibility that these areas might also be involved in controlling shifts of spatial attention. The attention mapping in the present study involved shifting attention to a new cued location every $3 \mathrm{~s}$.

Yantis et al. (2002) described an area within the left IPS that was active during a period of sustained attention to the right visual field, indicating that it codes for the current location of the focus of attention. The Talairach coordinates of this left IPS activation place it near IPS1 (4 to 6-mm difference between the two areas). Kastner et al. (1999) also described a left IPS activation during maintained attention to the right visual hemifield that is very close to IPS1. Unlike IPS1 and IPS2, the region described by Kastner et al. was reported to respond strongly to visual stimulation. However, the peripherally presented complex colored images used in the Kastner et al. study differed in several respects from the centrally presented monochromatic checkerboard visual stimuli used in the present study. Corbetta et al. (2000) identified multiple activations in 


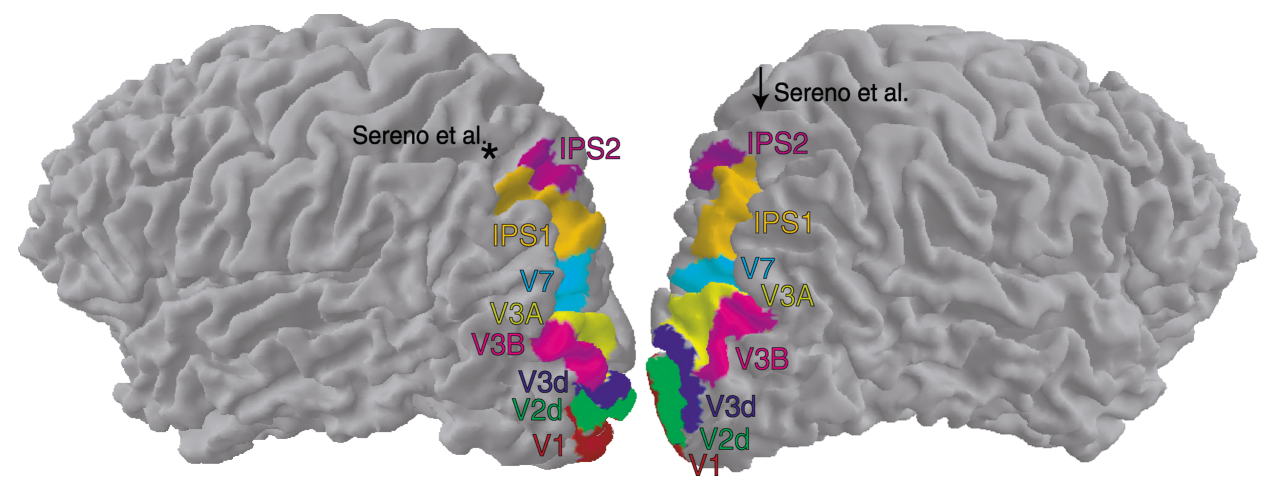

FIG. 10. Locations of topographic cortical areas. Left and right hemispheres are rotated $25^{\circ}$ from the lateral view to display more of the posterior surfaces. Topographically organized cortical areas tile the cortex continuously from V1 well into posterior parietal cortex. Asterisk: location of the Talairach coordinates corresponding to the center of the map reported in Sereno et al. (2000) in the left hemisphere. Arrow: location of the Sereno et al. map in the right hemisphere (not visible from this viewpoint). In both hemispheres of this subject (MAS), the center of the Sereno et al. map lies well outside the boundaries of IPS1 and IPS2. ventral, anterior, and posterior IPS associated with voluntary orienting of attention, and the ventral and anterior activations also exhibited maintained activity during a period of sustained attention. IPS2 is nearest to the posterior IPS activation identified by Corbetta et al. (2000). Tootell et al. (1998) reported maps of spatial attention in many extrastriate visual areas including V3d, V3A, and V7 but did not observe attention maps in more anterior parietal areas. They speculated that the absence of parietal activity in their attention task was due to the fact that the task required long periods of maintained attention and only infrequent switching of attention to new locations. Although the attention-mapping procedure used in the present study required frequent switching of attention, we have also demonstrated sustained activity in IPS1 and IPS2 during periods of maintained attention (Silver et al. 2003).

Hopfinger et al. (2000) used an event-related experimental design to dissociate fMRI responses related to switching of the focus of spatial attention (cue-related) and signals corresponding to subsequent processing of visual stimuli presented within the focus of attention (target-related) and reported cue-selective responses in many areas, including the IPS and the superior parietal lobule. However, the Talairach coordinates for the IPS activations reported in Hopfinger et al. (2000) are lateral to the IPS in all 8 hemispheres in our sample. In contrast, IPS1 and IPS 2 tend to be located on the medial bank of the IPS (Figs. 3 and 4). Thus it is unlikely that the nonlateralized cue-related activity reported by Hopfinger et al. (2000) corresponds to the lateralized topographic maps in IPS1 and IPS2.

\section{Topographic cortical areas in monkey and human PPC}

Monkey parietal cortex contains multiple functionally and anatomically defined areas that are believed to be functionally specialized for sensory attention, motor intention, and decision making (Andersen and Buneo 2002; Colby and Goldberg 1999; Glimcher 2003). Relatively little work, however, has been done to characterize the topographic organization of these cortical areas. One electrophysiological study mapped the visual receptive fields of neurons in the lateral intraparietal area (LIP) of anesthetized animals (Blatt et al. 1990). This study reported that LIP neurons preferentially responded to contralateral visual field locations. A second study of LIP (Ben Hamed et al. 2001), in awake monkeys performing a passive fixation task, reported a topographic representation of the contralateral visual field. The lower visual field was represented anteriorly, and the upper visual field was represented posteriorly. This visual field orientation in macaque area LIP was similar to that in our IPS2. However, visual field orientation, by itself, is only circumstantial evidence for a possible functional homology between macaque LIP and human IPS2. Moreover, a third study of monkey LIP (Platt and Glimcher 1998), in awake animals performing a saccade task, reported no evidence of laterality.

Sereno et al. (2001) described a topographic map in human PPC with a contralateral hemifield representation. The map was evident in a variety of tasks, with the "monitor return" task being most similar to the attention-mapping task used in the present study. The map described by Sereno et al. had a visual field orientation most similar to that of IPS1 (and opposite to that of IPS2) - that is, the lower visual field was represented ventrally/posteriorly, and the upper visual field was represented dorsally/anteriorly. However, the reported Talairach coordinates of the center of the posterior parietal map in the Sereno et al. study place it $15 \mathrm{~mm}$ anterior and $13 \mathrm{~mm}$ dorsal to the center of IPS1 (Fig. 10). Although the eccentricities used in the present study $\left(0.75-2.25^{\circ}\right)$ do not overlap with those used by Sereno et al. $\left(12-15^{\circ}\right)$, they also carried out a control task in which the eccentricities were randomized over a range of 3 to $15^{\circ}$. They reported that the map was unchanged by increasing the range of eccentricities, arguing against the possibility that IPS 1 and the Sereno et al. map represent different visual field representations of the same cortical map. Several other groups have preliminary reports (in abstract form) of topographic maps in human parietal cortex (DeSimone et al. 2004; Huddleston and DeYoe 2004; Jack et al. 2004; Sereno et al. 2004). The relationship between these maps and IPS1 and IPS2 is not yet clear.

Finally, the companion paper (Schluppeck et al. 2005) reports multiple topographic maps in human posterior parietal cortex, revealed with a delayed saccade task. Two subjects participated in both the attention-mapping (present study) and delayed-saccade (Schluppeck et al. 2005) studies. For these subjects, the two tasks generated the same pattern of topographic activity within the intraparietal sulcus (Fig. 9 of Schluppeck et al. 2005).

\section{A C K N O W L E D G M E N T S}

The authors thank J. Larsson for gray matter segmentation and cortical flattening software, M. Fukui and B. Knappmeyer for assisting with eye movement data collection, D. Schluppeck for many helpful discussions, and A. Shenhav for technical assistance.

Present addresses: D. J. Heeger, Department of Psychology and Center for Neural Science, New York University, 6 Washington Place, Room 809, New York, NY 10003; D. Ress, Brown University, Department of Neuroscience, Box 1953, 190 Thayer Street, Providence, RI 02912. 


\section{G R A N T S}

This research was supported by National Eye Institute Grant RO1-EY11794, National Research Service Award F32 EY-14520, and a grant from the Seaver Foundation.

\section{R E F E R E N C E S}

Andersen RA and Buneo CA. Intentional maps in posterior parietal cortex. Annu Rev Neurosci 25: 189-220, 2002.

Bashinski HS and Bacharach VR. Enhancement of perceptual sensitivity as the result of selectively attending to spatial locations. Percept Psychophys 28: 241-248, 1980

Ben Hamed S, Duhamel J-R, Bremmer F, and Graf W. Representation of the visual field in the lateral intraparietal area of macaque monkeys: a quantitative receptive field analysis. Exp Brain Res 140: 127-144, 2001.

Bisley JW and Goldberg ME. Neuronal activity in the lateral intraparietal area and spatial attention. Science 299: 81-86, 2003.

Blatt GJ, Andersen RA, and Stoner GR. Visual receptive field organization and cortico-cortical connections of the lateral intraparietal area (area LIP) in the macaque. J Comp Neurol 299: 421-445, 1990.

Brefczynski JA and DeYoe EA. A physiological correlate of the "spotlight" of visual attention. Nat Neurosci 2: 370-374, 1999.

Colby CL and Goldberg ME. Space and attention in parietal cortex. Апnи Rev Neurosci 22: 319-349, 1999.

Corbetta M, Kincade JM, Ollinger JM, McAvoy MP, and Shulman GL. Voluntary orienting is dissociated from target detection in human posterior parietal cortex. Nat Neurosci 3: 292-297, 2000.

Corbetta M and Shulman GL. Control of goal-directed and stimulus-driven attention in the brain. Nat Rev Neurosci 3: 201-215, 2002.

DeSimone KD, Schneider KA, Pinsk MA, Norman K, and Kastner S. Response properties of a putative LIP area in human parietal cortex. Soc Neurosci Abstr 751.16, 2004.

DeYoe EA, Carman GJ, Bandettini P, Glickman S, Wieser J, Cox R, Miller D, and Neitz J. Mapping striate and extrastriate visual areas in human cerebral cortex. Proc Natl Acad Sci USA 93: 2382-2386, 1996.

Dougherty RF, Koch VM, Brewer AA, Fischer B, Modersitzki J, and Wandell BA. Visual field representations and locations of visual areas V1/2/3 in human visual cortex. J Vision 3: 586-598, 2003.

Dumoulin SO, Baker CL Jr, Hess RF, and Evans AC. Cortical specialization for processing first- and second-order motion. Cereb Cortex 13: 13751385, 2003.

Engel SA, Glover GH, and Wandell BA. Retinotopic organization in human visual cortex and the spatial precision of functional MRI. Cereb Cortex 7: 181-192, 1997.

Engel SA, Rumelhart DE, Wandell BA, Lee AT, Glover GH, Chichilnisky E-J, and Shadlen MN. fMRI of human visual cortex. Nature 369: 525, 1994.

Gandhi SP, Heeger DJ, and Boynton GM. Spatial attention affects brain activity in human primary visual cortex. Proc Natl Acad Sci USA 96: 3314-3319, 1999.

Glimcher PW. The neurobiology of visual-saccadic decision making. Annu Rev Neurosci 26: 133-179, 2003.

Glover GH. Simple analytic spiral k-space algorithm. Magn Res Med 42: 412-415, 1999.

Glover GH and Lai S. Self-navigated spiral fMRI: interleaved versus singleshot. Magn Res Med 39: 361-368, 1998.

Goldberg ME, Bisley J, Powell KD, Gottlieb J, and Kusunoki M. The role of the lateral intraparietal area of the monkey in the generation of saccades and visuospatial attention. Ann NY Acad Sci 956: 205-215, 2002.

Hopfinger JB, Buonocore MH, and Mangun GR. The neural mechanisms of top-down attentional control. Nat Neurosci 3: 284-291, 2000.

Huddleston WE and DeYoe EA. Two task-related topographic maps of visuospatial attention in human parietal cortex. Hum Brain Mapp Abstr TH49, 2004.

Intriligator $\mathbf{J}$ and Cavanaugh $\mathbf{P}$. The spatial resolution of visual attention. Cognit Psychol 43: 171-216, 2001.

Jack AI, Patel GH, Astafiev SV, Snyder AZ, McAvoy M, Shulman GL, and Corbetta M. Retinotopic specificity in human parietal, temporal, and frontal cortices. Soc Neurosci Abstr 717.3, 2004.

Janik JJ, Ropella KM, and DeYoe EA. Distortions of human retinotopy obtained with temporal phase mapped fMRI. Soc Neurosci Abstr 658.8, 2003.
Jenkinson M, Bannister P, Brady M, and Smith S. Improved optimization for the robust and accurate linear registration and motion correction of brain images. Neuroimage 17: 825-841, 2002.

Kastner S, Pinsk MA, De Weerd P, Desimone R, and Ungerleider LG. Increased activity in human visual cortex during directed attention in the absence of visual stimulation. Neuron 22: 751-761, 1999.

Kusunoki M, Gottlieb J, and Goldberg ME. The lateral intraparietal area as a salience map: the representation of abrupt onset, stimulus motion, and task relevance. Vision Res 40: 1459-1468, 2000.

Larsson J. Imaging Vision: Functional Mapping of Intermediate Visual Processes in Man (PhD Thesis). Stockholm, Sweden: Karolinska Institute, 2001.

Martinez A, Anllo-Vento L, Sereno MI, Frank LR, Buxton RB, Dubowitz DJ, Wong EC, Hinrichs H, Heinze HJ, and Hillyard SA. Involvement of striate and extrastriate visual cortical areas in spatial attention. Nat Neurosci 2: 364-369, 1999 .

McMains SA and Somers DC. Multiple spotlights of attentional selection in human visual cortex. Neuron 42: 677-686, 2004.

Mesulam M-M. Spatial attention and neglect: parietal, frontal and cingulate contributions to the mental representation and attentional targeting of salient extrapersonal events. Philos Trans R Soc Lond B Biol Sci 354: 1325-1346, 1999.

Müller NG and Kleinschmidt A. The attentional "spotlight's" penumbra: center-surround modulation in striate cortex. Neuroreport 15: 977-980, 2004.

Nestares $\mathrm{O}$ and Heeger DJ. Robust multiresolution alignment of MRI brain volumes. Magn Res Med 43: 705-715, 2000.

Pashler HE. The Psychology of Attention. Cambridge, MA: MIT Press, 1998

Platt ML and Glimcher PW. Response fields of intraparietal neurons quantified with multiple saccadic targets. Exp Brain Res 121: 65-75, 1998.

Posner MI. Orienting of attention. Q J Exp Psychol 32: 3-25, 1980.

Posner MI, Walker JA, Friedrich FJ, and Rafal RD. Effects of parietal injury on covert orienting of attention. J Neurosci 4: 1863-1874, 1984.

Press WA, Brewer AA, Dougherty RF, Wade AR, and Wandell BA. Visual areas and spatial summation in human visual cortex. Vision Res 41: 13211332, 2001.

Ress D, Backus BT, and Heeger DJ. Activity in primary visual cortex predicts performance in a visual detection task. Nat Neurosci 3: 940-945, 2000.

Ress D and Heeger DJ. Neuronal correlates of perception in early visual cortex. Nat Neurosci 6: 414-420, 2003.

Sasaki Y, Hadjikhani N, Fischl B, Liu AK, Marrett S, Dale AM, and Tootell RBH. Local and global attention are mapped retinotopically in human occipital cortex. Proc Natl Acad Sci USA 98: 2077-2082, 2001.

Schluppeck D, Glimcher P, and Heeger DJ. Topographic organization for delayed saccades in human posterior parietal cortex. J Neurophysiol 94: 1371-1383, 2005.

Schneider KA, Richter MC, and Kastner S. Retinotopic organization and functional subdivisions of the human lateral geniculate nucleus: a highresolution functional magnetic resonance imaging study. J Neurosci 24: 8975-8985, 2004.

Sereno MI, Dale AM, Reppas JB, Kwong KK, Belliveau JW, Brady TJ, Rosen BR, and Tootell RBH. Borders of multiple visual areas in humans revealed by functional MRI. Science 268: 889-893, 1995.

Sereno MI, Huang R, Saygin A, Filimon F, and Hagler D. Retinotopy of human cortex using phase-encoded video. Soc Neurosci Abstr 709.7, 2004.

Sereno MI, Pitzalis S, and Martinez A. Mapping of contralateral space in retinotopic coordinates by a parietal cortical area in humans. Science 294: 1350-1354, 2001.

Shulman GL, Ollinger JM, Akbudak E, Conturo TE, Snyder AZ, Petersen SE, and Corbetta M. Areas involved in encoding and applying directional expectations to moving objects. J Neurosci 19: 9480-9496, 1999.

Silver MA, Ress D, and Heeger DJ. Neural correlates of sustained attention in parietal and primary visual cortex. Soc Neurosci Abstr 873.5, 2003.

Silver MA, Ress D, and Heeger DJ. Retinotopic maps of visual spatial attention in human parietal cortex. Cogn Neurosci Soc Abstr 11, 2004a.

Silver MA, Shenhav A, Heeger DJ, and D'Esposito M. Cholinergic enhancement of top-down visual spatial attention in humans. Soc Neurosci Abstr 480.2, 2004b.

Smith AM, Lewis BK, Ruttimann UE, Ye FQ, Sinnwell TM, Yang Y, Duyn JH, and Frank JA. Investigation of low frequency drift in fMRI signal. Neuroimage 9: 526-533, 1999. 
Smith AT, Greenlee MW, Singh KD, Kraemer FM, and Hennig J. The processing of first- and second-order motion in human visual cortex assessed by functional magnetic resonance imaging (fMRI). J Neurosci 18: $3816-$ 3830, 1998

Smith AT, Singh KD, and Greenlee MW. Attentional suppression of activity in the human visual cortex. Neuroreport 11: 271-277, 2000.

Sokal RR and Rohlf FJ. Biometry: The Principles and Practice of Statistics in Biological Research. New York: Freeman, 1995.

Stone $\mathbf{J}$ and Johnston E. The topography of primate retina: a study of the human, bushbaby, and New- and Old-World monkeys. J Comp Neurol 196: 205-223, 1981.

Teo PC, Sapiro G, and Wandell BA. Creating connected representations of cortical gray matter for functional MRI visualization. IEEE Trans Med Imaging 16: 852-863, 1997.

Tootell RBH and Hadjikhani N. Where is "dorsal V4" in human visual cortex? Retinotopic, topographic and functional evidence. Cereb Cortex 11: 298-311, 2001
Tootell RBH, Hadjikhani N, Hall EK, Marrett S, Vanduffel W, Vaughan JT, and Dale AM. The retinotopy of visual spatial attention. Neuron 21: 1409-1422, 1998.

Tootell RBH, Mendola JD, Hadjikhani NK, Ledden PJ, Liu AK, Reppas JB, Sereno MI, and Dale AM. Functional analysis of V3A and related areas in human visual cortex. J Neurosci 17: 7060-7078, 1997.

Wandell BA, Brewer AA, and Dougherty RF. Visual field map clusters in human cortex. Philos Trans R Soc Lond B Biol Sci 360: 10.1098/rstb. 2005.1628, 2005.

Wandell BA, Chial S, and Backus BT. Visualization and measurement of the cortical surface. J Cog Neurosci 12: 739-752, 2000.

Yantis S, Schwarzbach J, Serences JT, Carlson RL, Steinmetz MA, Pekar JJ, and Courtney SM. Transient neural activity in human parietal cortex during spatial attention shifts. Nat Neurosci 5: 995-1002, 2002.

Zarahn E, Aguirre GK, and D'Esposito M. Empricial analyses of BOLD fMRI statistics. I. Spatially unsmoothed data collected under null-hypothesis conditions. Neuroimage 5: 179-197, 1997. 